\title{
Una experiencia de integración de la Formación Sanitaria Especializada con la Universitaria de Posgrado: Entrenamiento por etapas en cirugía laparoscópica.
}

\section{An experience of integration of specialized medical training with university postgraduate learning: Stage-based training in laparoscopic surgery.}

\begin{abstract}
Diego Flores-Funes ${ }^{1}$, Enrique Pellicer-Franco ${ }^{2}$, Benito Flores-Pastor ${ }^{3}$, Matilde Moreno Cascales $^{4}$, Miguel Ángel Fernández-Villacañas-Marín ${ }^{5,}$ José Luis Aguayo-Albasini $^{6 *}$

${ }^{1}$ Facultativo Especialista de Área Cirugía General. Profesor Colaborador Docente. Hospital General Universitario Morales Meseguer. CEUEM. Murcia diego.ff90@gmail.com.

2 Facultativo Especialista de Área Cirugía General. Profesor Asociado. Hospital General Universitario Morales Meseguer. CEUEM. Murcia e.pellicerfranco@gmail.com.

3 Facultativo Especialista de Área Cirugía General. Profesor Asociado. Hospital General Universitario Morales Meseguer. CEUEM. Murcia benitomanuel.flores@um.es.

4 Profesor Titular de Universidad. Área de Anatomía. Universidad de Murcia. CEUEM. mmc@um.es.

${ }^{5}$ Profesor Titular de Universidad. Área de Anatomía. Universidad de Murcia mafvm@um.es.

${ }^{6}$ Catedrático de Universidad. Área de Cirugía. Universidad de Murcia. Jefe del Servicio de Cirugía General del Hospital General Universitario Morales Meseguer. Murcia (España). CEUEM. IMIB-Arrixaca. jlaguayo@um.es. ORCID ID https://orcid.org/0000-0002-2979-7047

* Correspondencia: jlaguayo@um.es
\end{abstract}

Recibido: 7/11/2020; Aceptado: 27/11/2020; Publicado: 27/11/2020

Resumen: Antecedentes: Diseño de un modelo de simulación para formación en cirugía laparoscópica. Métodos: Dentro del Máster Anatomía Aplicada a la Clínica se implementaron tres etapas de formación progresiva en médicos residentes. La etapa 1 se realizó en simulador con material no biológico: manejo de objetos, disección con pinza y tijera, y sutura laparoscópica. La etapa 2 utilizó material biológico (tubo digestivo en fresco) en simulador, para la práctica de sección intestinal y anastomosis intracorpórea. En la etapa 3 se realizaron técnicas laparoscópicas en cadáver en fresco (apendicectomía, colecistectomía, apertura de la transcavidad epiploica, hemicolectomías derecha e izquierda). Se añadió una encuesta de satisfacción a los participantes de la actividad. Resultados: El programa se impartió a 6 residentes de Cirugía General. Todos completaron los ejercicios de las etapas 1 y 2. En la etapa 3 se pudo realizar el neumoperitoneo sin dificultad y los tejidos presentaron una textura adecuada. Los residentes de primer año completaron la apendicectomía y la colecistectomía, pero procedimientos más complejos requirieron residentes con más experiencia. Los participantes encuestados reflejaron que el programa es adecuado y útil para el entrenamiento en laparoscopia. Conclusiones: El modelo propuesto es reproducible y adecuado en adquisición de competencias básicas en cirugía laparoscópica.

Palabras Clave: Laparoscopia. Residentes. Educación quirúrgica. Habilidades técnicas. Simulación. 


\begin{abstract}
Background: Design of a simulation model training in laparoscopic surgery for surgical residents. Methods: Three stages of progressive training were programmed within a Postgraduate Degree in Clinical Anatomy. Stage 1 was performed in a box-trainer with synthetic materials: managing small objects, dissection with clamp and scissors, and laparoscopic intracorporeal suture. Stage 2 used biological material (fresh digestive tract from a human corpse) in box-trainer, practicing section and intracorporeal anastomosis. In stage 3, laparoscopy was performed on a fresh corpse (appendectomy, cholecystectomy, lesser sac opening, right and left colectomy). A satisfaction survey was carried out to the participants. Results: Some six General and Digestive Surgery residents took the program. All of them completed the stage 1 and 2 exercises. In stage 3, the pneumoperitoneum could be performed without any complications, and tissues presented an adequate texture. First-year residents completed appendectomy and cholecystectomy, but more complex procedures required more experienced residents. The participants reflected that the program is adequate and useful to gain basic skills in laparoscopy. Conclusions: The proposed model is reproducible and adequate in acquisition of basic skills in laparoscopic surgery.
\end{abstract}

Keywords: Laparoscopy. Resident. Surgical education. Technical skills. Simulation.

\title{
1. Introducción.
}

La mayoría de los procedimientos quirúrgicos abdominales pueden realizarse mediante abordaje laparoscópico y en la actualidad no se cuestionan sus ventajas en cuanto a confort postoperatorio, recuperación del paciente y resultado estético. Por ello, la formación en este tipo de cirugía debe comenzar desde el período de residencia (1). Sin embargo, el principal problema de su entrenamiento es la larga curva de aprendizaje y los errores y complicaciones que pueden surgir al practicar directamente sobre pacientes (2). De ahí nace la simulación quirúrgica como una herramienta ideal para la adquisición de destrezas básicas en este tipo de cirugía (3).

En España, la Orden SSI/81/2017 (4) refuerza la importancia de los procedimientos de formación basados en la simulación clínica, al indicar que los Centros sanitarios deben favorecer la utilización de la simulación como paso previo a realizar técnicas en pacientes reales, y que se deben incluir actividades simuladas en los convenios con Centros educativos.

Presentamos un programa de formación en cirugía laparoscópica con un diseño modular basado en la realización de talleres por etapas, dirigido a residentes de Cirugía General y del Aparato Digestivo. Estas sesiones están integradas dentro del programa del Máster Universitario en Anatomía Aplicada a la Clínica de la Universidad de Murcia, coordinado por profesores de Anatomía en colaboración con cirujanos del Hospital Universitario Morales Meseguer de Murcia (España).

\section{Metodología.}

El modelo tiene un diseño en tres etapas, cada una con un creciente nivel de complejidad que el alumno debe superar para poder enfrentarse a la fase siguiente. Cada etapa engloba una serie de ejercicios para el aprendizaje de competencias básicas en cirugía laparoscópica y están dirigidos a residentes de $1^{\underline{0}-3^{0}}$ año. En cada sesión hay 2-3 alumnos, que son supervisados por dos cirujanos con más de 20 años de experiencia en cirugía laparoscópica. 
La infraestructura utilizada ha sido un entrenador laparoscópico (modelo MED3X $\odot$ de la casa Medical Simulator Spain), con orificios para trócares, cámara y pantalla incorporadas, 2 portaagujas laparoscópicos, y material fungible: trócares de 5, 11 y $12 \mathrm{~mm}$, disectores, tijeras y pinzas. Para la tercera etapa también se ha utilizado una torre laparoscópica estándar con cámara y óptica de luz fría, una bomba de insuflación de $\mathrm{CO}_{2}$ y un sistema de aspiración.

En la primera etapa se utiliza el simulador laparoscópico junto con modelos sintéticos que creamos a partir de material simple (que se puede comprar en cualquier tienda de manualidades, figura 1). Se plantearon 5 ejercicios: En el primero, el alumno debe introducir cuentas de collar en las ranuras de un bote, cada ranura de un color diferente, para adquirir destreza en el manejo de las pinzas y en la bidimensionalidad. En el segundo ejercicio, ideal para aprender a utilizar el disector y las dos manos, consiste en pasar un hilo por cáncamos atornillados a una tabla de madera, y realizar un nudo intracorpóreo. En el tercer ejercicio se practica la sutura intracorpórea, teniendo que realizar una sutura simple y continua sobre un modelo de sutura creado a partir de un apósito hidrocoloide sobre gasas. El cuarto ejercicio, algo más complejo, consiste en disecar debajo de un apósito, un globo de agua envuelto en gasa que asemeja un vaso sanguíneo, y ligarlo con un hilo de sutura. Y por último, en el quinto ejercicio se confecciona una anastomosis termino-terminal entre dos globos desinflados, con una sutura continua en la cara posterior y otra en la cara anterior.

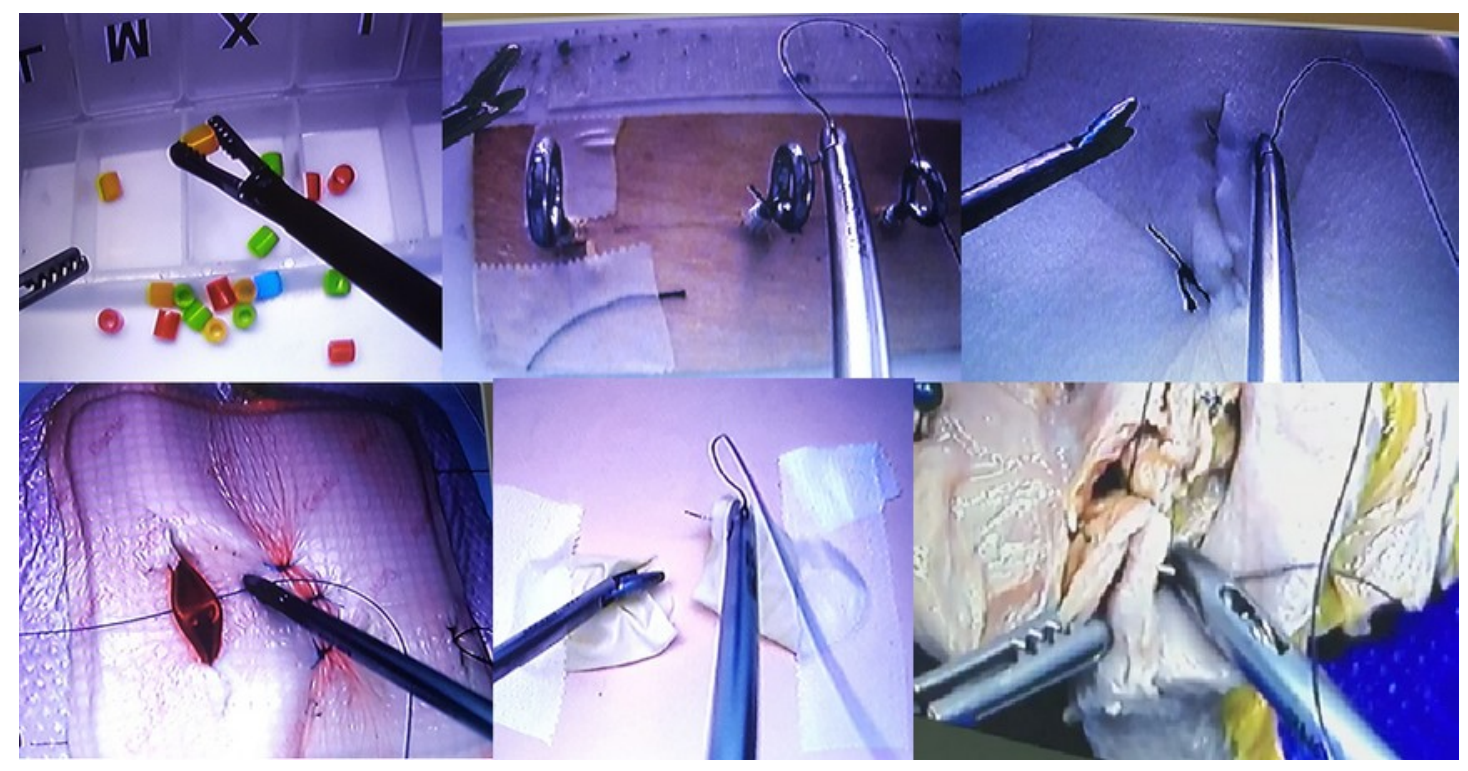

Figura 1. Fotografías de los ejercicios de las etapas 1 y 2.

En la etapa 2 se emplea también el simulador laparoscópico, pero utilizando tejido fresco de cadáver sin fijar, más específicamente el tubo digestivo (estómago, intestino delgado y grueso). En el estómago se realiza una apertura antro-pilórica y luego una piloroplastia. Sobre el intestino, previa sección, se practica una anastomosis términoterminal seguida de otra latero-lateral.

En la etapa 3 (figura 2) llegamos a la fase más compleja, que es la realización de cirugía laparoscópica sobre cadáver sin fijar. Aquí, los alumnos se turnaron según su nivel de experiencia para realizar (con la ayuda de disección fría con tijera y endoclips para controlar vasos) los siguientes procedimientos: apendicectomía, colecistectomía, apertura gástrica y sutura de la misma, anastomosis intestinal manual, y la disección 
de los vasos y el despegamiento del colon derecho e izquierdo (sin llegar a realizar la colectomía).

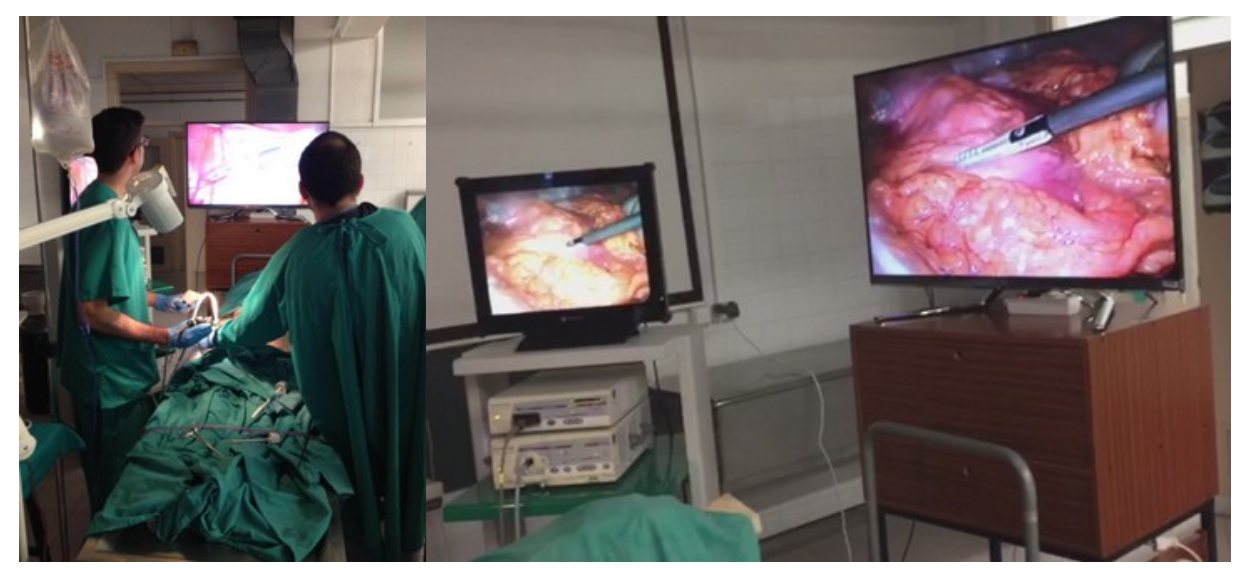

Figura 2. Fotografía de la etapa 3. A la izquierda, posición de los alumnos a la hora de realizar los ejercicios. A la derecha, imagen de los monitores de laparoscopia en cadáver.

Tras la terminación de la actividad, los participantes completaron una encuesta anonimizada evaluando mediante una escala Likert cuatro items: la utilidad, los recursos empleados, el equipo docente, y la satisfacción general de cada una de las etapas.

\section{Resultados}

La acción docente fue impartida a 6 residentes de Cirugía General y del Aparato Digestivo de primer y segundo año, con una duración total de 16 horas por alumno (4 horas en la etapa 1, 4 horas en la etapa 2, y 8 horas en la etapa 3). Todos los alumnos completaron los ejercicios de las 2 primeras etapas sin ninguna dificultad. La etapa 3 fue la más compleja técnicamente y en cuanto a infraestructura. La creación del neumoperitoneo en el cadáver pudo hacerse sin ningún problema. Los tejidos del cadáver sin fijar presentaron una textura adecuada para poder realizar disección fría y roma, asemejándose bastante a la del vivo (aunque sin emularla a la perfección). La apendicectomía y la colecistectomía eran realizables incluso por los residentes de menor año, pero las técnicas de sutura intestinal y disección vascular fueron claramente más asequibles a los residentes mayores.

En cuanto a las encuestas, encontramos puntuaciones de 4 y de 5 en todos los ítems, lo que confirmó una alta satisfacción con las actividades realizadas. La etapa 1 fue la menos valorada, sobre todo en lo que se refiere a recursos empleados, con una media de 4,3 (figura 3). El equipo docente fue la parte mejor valorada en esta etapa, con una media de 4,8 .

La etapa 2 fue algo mejor valorada (figura 4), siendo de nuevo los recursos empleados lo peor considerado, con un 4,7, mientras que el equipo docente recibió la máxima puntuación.

La etapa 3 (figura 5) fue la mejor valorada de todas, con todos los ítems puntuados con una magnitud cercana al 5. La utilidad fue lo peor valorado por los alumnos, aunque con una alta puntuación de 4,8. 


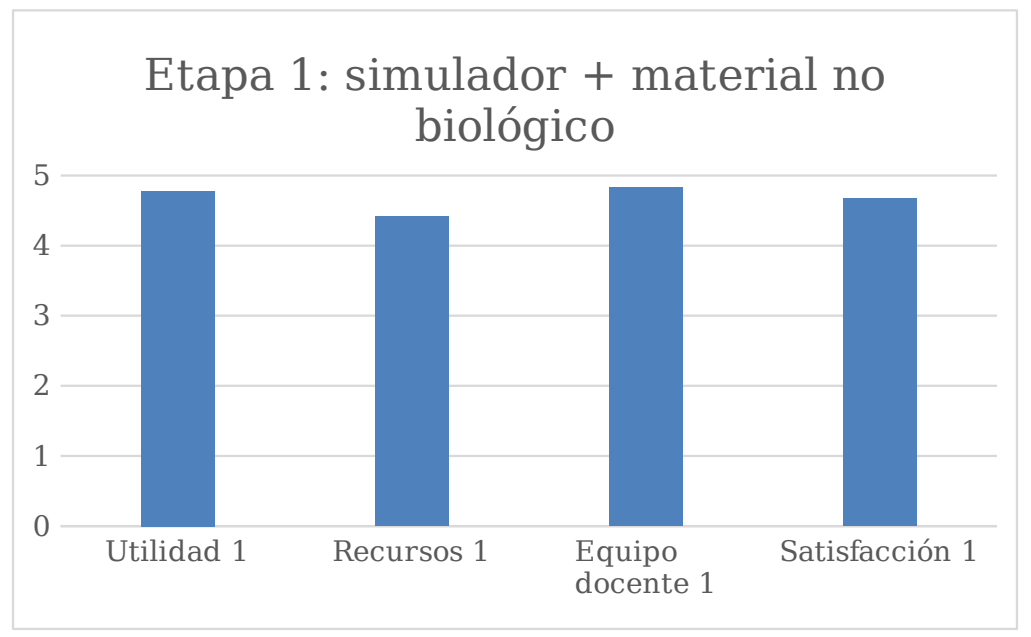

Figura 3. Gráfico de barras de los ítems de la encuesta de satisfacción de la etapa 1: simulador y material no biológico.

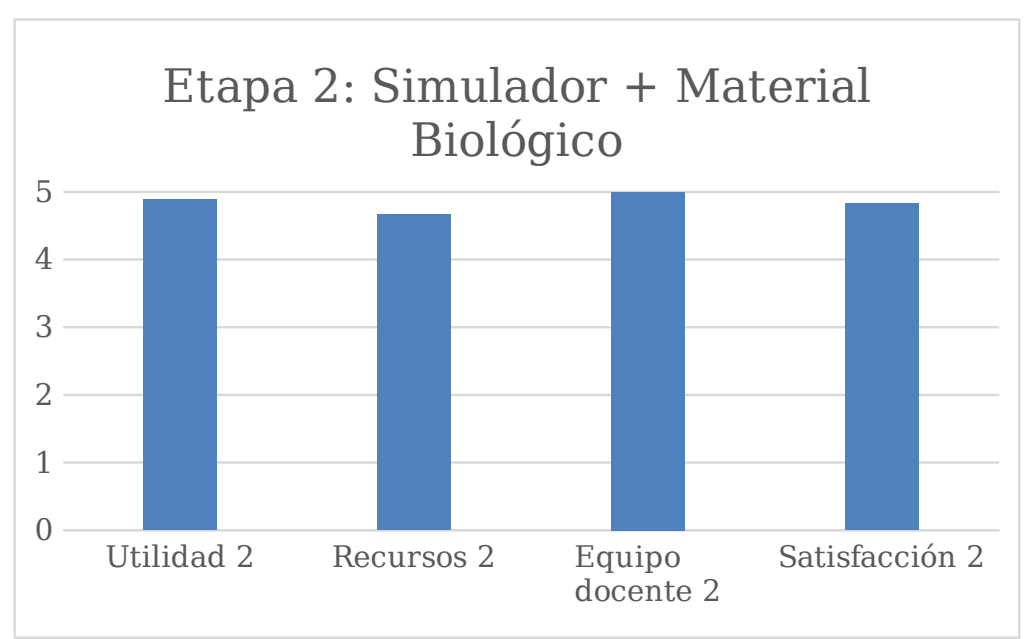

Figura 4. Gráfico de barras de los ítems de la encuesta de satisfacción de la etapa 2: simulador y material biológico.

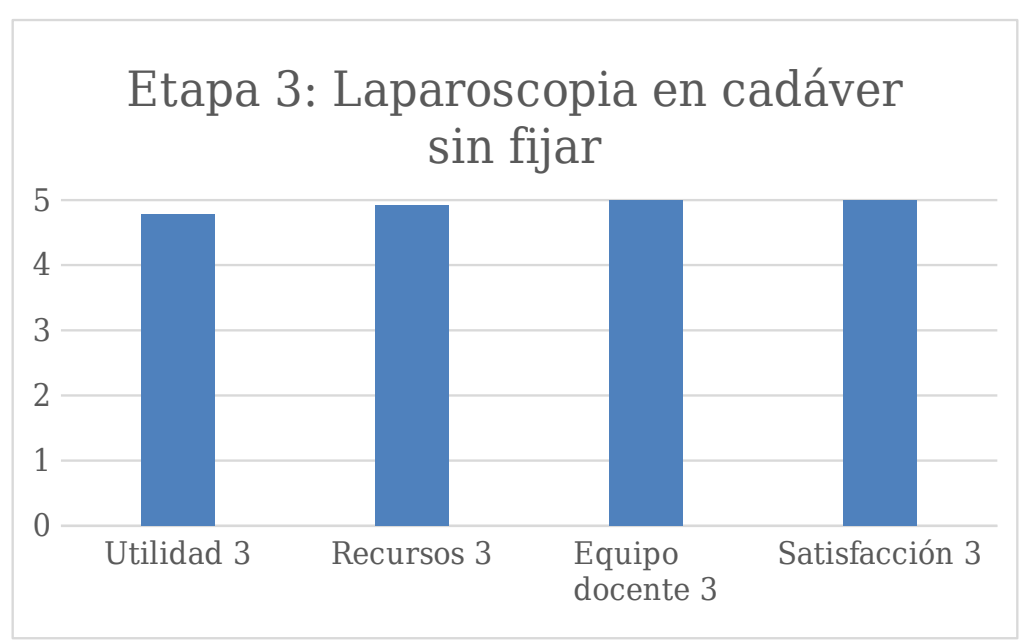

Figura 5. Gráfico de barras de los ítems de la encuesta de satisfacción de la etapa 3: laparoscopia en cadáver sin fijar. 


\section{Discusión}

Este programa de formación surgió a raíz del contacto entre expertos clínicos (con responsabilidad en formación de Médicos Internos Residentes) y profesores de Anatomía Humana, dentro del Máster de Anatomía Aplicada a la Clínica de la Universidad de Murcia. Aunque no sea competencia directa de las Universidades la formación de médicos residentes, su contribución en este tipo de actividades es esencial para complementar la formación en competencias que el ámbito hospitalario no puede ofrecer. Ello se remarca en la adquisición de habilidades quirúrgicas que de otra manera se aprenderían mediante la observación y la participación directa en cirugía de pacientes, lo cual puede enlentecer el proceso formativo o suponer un conflicto ético (2).

Este modelo de formación por etapas ha sido diseñado por cirujanos con muchos años de experiencia en formación de residentes quirúrgicos, pero además ha incluido la opinión de los residentes, lo cual es fundamental para poder atender las competencias básicas en cirugía laparoscópica que deben adquirir durante su formación. El material utilizado, a excepción del simulador y los portaagujas laparoscópicos, fue de bajo coste dado que era fungible y reutilizado tras ser usado en quirófano, y los modelos fueron creados con material barato y fácilmente accesible. Por ello, el programa podría perfectamente reproducirse en cualquier Facultad de Medicina con una sala de disección, no requiriendo grandes inversiones para su implementación. Los autores pensamos que lo ideal sería que en el futuro este tipo de actividades fueran obligatorias y estuvieran integradas dentro del programa de formación de residentes quirúrgicos.

Es cierto que no existe suficiente evidencia de que los modelos de simulación laparoscópica basados en cajas reduzcan la morbimortalidad de los pacientes quirúrgicos, pero existen algunos estudios que sugieren que mejoran las habilidades y reducen el tiempo operatorio respecto a los cirujanos entrenados de manera tradicional con pacientes reales $(5,6)$, sin mencionar, como se ha comentado anteriormente, el conflicto ético que puede suponer el entrenamiento directo en pacientes.

Por otro lado, el modelo de cadáver sin fijar ha sido descrito anteriormente en diferentes estudios. Sharma y cols. (7) realizaron un ensayo clínico en residentes junior, aleatorizando dos grupos con y sin entrenamiento en cadáver. Encontraron diferencias significativas al evaluar a cada grupo mediante la Global Operative Assessment of Laparoscopic Skills scale (GOALS), escala validada para la evaluación de habilidades en cirugía laparoscópica (8). En otro estudio, Palter y Grantcharov (9) llevaron a cabo un programa semejante al presentado en este trabajo, con una primera etapa en simulador y una segunda en cadáver, mostrando unos mejores conocimientos técnicos y mayor habilidad en quienes recibieron dicho entrenamiento. Aunque en nuestro trabajo no tengamos datos objetivos de la adquisición de competencias quirúrgicas, la satisfacción reflejada por los alumnos sugiere que es una actividad de su agrado y que la consideran importante y útil para su formación.

Este modelo tiene algunas limitaciones que nos gustaría comentar, de cara a poder ser mejorado en el futuro. En primer lugar, no disponemos de infraestructura y dispositivos quirúrgicos más específicos o avanzados, como electrocoagulación o endograpadoras. Además, tenemos dudas de que la diatermia pueda utilizarse en un cadáver, por lo que el manejo de este instrumental no ha podido realizarse. Por otra parte, el cadáver tiene la sangre coagulada, por lo que no sangra y el alumno no puede practicar el control de la hemorragia durante la cirugía laparoscópica. Y 
también destacamos que, según la técnica ensayada, había que ir cerrando determinados orificios de los trócares y abriendo otros nuevos, con lo que frecuentemente se perdía el gas del neumoperitoneo (aunque se mantenía dentro de la cavidad peritoneal y no dificultaba la práctica), lo que incomodaba la técnica con el paso del tiempo. Finalmente, el bajo número de participantes hace que los resultados obtenidos y las conclusiones resultantes precisen confirmación en estudios más amplios.

\section{Conclusiones.}

- El modelo propuesto es factible para la adquisición de competencias básicas en cirugía laparoscópica, si bien con las limitaciones comentadas.

- Son importantes las acciones y esfuerzos conjuntos entre los Centros hospitalarios y las Facultades de Medicina, posibilidad que muchas veces se olvidan o rechazan en la formación de postgrado.

- Finalmente, son necesarias evaluaciones objetivas de este modelo, utilizando escalas validadas como la GOALS, para asegurar su validez.

Material suplementario: No hay

Financiación: No ha habido financiación

Agradecimientos: A los médicos residentes que participaron y aprendieron con esta acción.

Declaración de conflicto de intereses: Los autores declaran no tener conflicto de intereses.

\section{Referencias}

1. Quick JA, Kudav V, Doty J, Crane M, Bukoski AD, Bennett, BJ, Barnes SL. Surgical resident technical skill self-evaluation: increased precision with training progression. J Surg Res. 2017;218:144-149. http://doi.org/10.1016/j.jss.2017.05.070.

2. Meaghe AP, Yang S, Li S. Is it right to ignore learning-curve patients? Laparoscopic colorectal trials. ANZJ Surg. 2017;87(11):898-902. http://doi.org/10.1111/ans.14070

3. Shetty S, Zevin B, Grantcharov TP, Roberts KE, Duffy AJ. Perceptions, Training Experiences, and Preferences of Surgical Residents Toward Laparoscopic Simulation Training: A Resident Survey. J Surg Ed. 2014;71(5):727-733. http://doi.org/10.1016/j.jsurg.2014.01.006.

4. Orden SSI/81/2017, de 19 de enero, por la que se publica el Acuerdo de la Comisión de Recursos Humanos del Sistema Nacional de Salud, por el que se aprueba el protocolo mediante el que se determinan pautas básicas destinadas a asegurar y proteger el derecho a la intimidad del paciente por los alumnos y residentes en Ciencias de la Salud. BOE 31, de 6 de febrero de 2017. https://www.boe.es/eli/es/o/2017/01/19/ssi81

5. Fu S, Liu X, Zhou L, Zhou M, Wang L. Applied Research on Laparoscopic Simulator in the Resident Surgical Laparoscopic Operation Technical Training. Indian J Surg. 2017;79(4): 288-293. http://doi.org/10.1007/s12262-016-1468-z

6. Gurusamy KS, Nagendran M, Toon CD, Davidson BR. Laparoscopic surgical box model training for surgical trainees with limited prior laparoscopic experience. Cochrane Database Syst Rev. 2014; 1(3): CD010478. http://doi.org/ 10.1002/14651858.CD010478.pub2.

7. Sharma M. Macafee D, Horgan AF. (2013). Basic laparoscopic skills training using fresh frozen cadaver: a randomized controlled trial. Am J Surg. 2013;206(1): 23-31. http://doi.org/10.1016/j.amjsurg.2012.10.037 
8. Vassiliou MC, Feldman L S, Andrew C.G, Bergman S, Leffondré K, Stanbridge D, Fried GM. A global assessment tool for evaluation of intraoperative laparoscopic skills. Am J Surg. 2005;190(1), 107-113. http://doi.org/10.1016/j.amjsurg.2005.04.004.

9. Palter VN, Grantcharov TP. (2012). Development and Validation of a Comprehensive Curriculum to Teach an Advanced Minimally Invasive Procedure. Ann Surg. 2012;256(1), 25-32. http://doi.org/10.1097/SLA.0b013e318258f5aa.

(C) 2020 por los autores. Enviado para su publicación en acceso abierto bajo los términos y condiciones de la licencia Creative Commons Attribution (CC BY) (http://creativecommons.org/licenses/by/4.0/). 